\title{
Ochratoxin A: Comparative pharmacokinetics and toxicological implications (experimental and domestic animals and humans)
}

\author{
DANIEL R. DIETRICH, ALEXANDRA H. HEUSSNER, \& EVELYN O’BRIEN
}

Department of Environmental and Human Toxicology, University of Konstanz, Germany

\begin{abstract}
The causal factors for the species- and sex-differences associated with ochratoxin-mediated toxicity remain unclear. Variations in kinetic parameters may play a major role in explaining these differences, however, discrepancies and inaccuracies in the toxicokinetics reported in the literature for various species, make comparison and hence the extrapolation to the human situation impossible. The one- and two-compartment open models currently proposed may be insufficient to enable an accurate representation of the actual situation in vivo. It is likely that at least three if not four compartments must be assumed to account for the reported effects. The application of such models to existing raw data would most likely provide for a more accurate base set of toxicokinetic data and contribute to a more accurate human risk assessment. Possible explanations for the reported inconsistencies and their impact on the proposed mechanism(s) of action of OTA and risk assessment are discussed.
\end{abstract}

Keywords: Ochratoxin $A$, toxicokinetics, modeling, risk assessment

\section{Introduction}

Numerous kinetic experiments with different species aimed at the provision of a fundamental understanding of the mechanism(s) underlying the acute, subchronic and chronic toxicity and carcinogenicity of ochratoxin A (OTA) have been carried out in the past decades. Indeed, one of the primary aims of these studies was to achieve a better basis for human risk assessment, especially in the light of the epidemiological data suggesting an association of OTA with the aetiology of diseases in humans, e.g. Balkan Endemic Nephropathy (BEN) and urothelial tumours (UT) and pigs, e.g. Danish Porcine Nephropathy (DPN). However, stark species- and sex-differences observed in chronic/carcinogenicity studies, especially those in two-year rodent bioassays (Bendele et al. 1985, Boorman 1989), have proven to be more enigmatic than enlightening and thus rather problematic for use as a scientific basis for human cancer risk assessment. However, these two-year rodent bioassays do not and cannot provide data relevant to the generation of the nephropathy/ progressive renal fibrosis assumed to be associated with chronic exposure to ochratoxin $\mathrm{A}$ in humans and pigs. Furthermore, profound differences in OTA distribution and elimination as well as in OTA sensitivity, between rodent and humans, as discussed below, suggest that the assessment or safety factor approach currently proposed by the IPCS (IPCS/ WHO 2005) may not suffice for the development of a reliable exposure dose-response model and consequently for a tolerable daily intake.

\section{Kinetic studies}

Several important kinetic parameters were determined primarily using single applications of orally (gavage) or intraveneously (i.v.) administered OTA (see Tables I and II). A principal problem in the determination of the kinetic parameters in most studies to date is that the number of time-points in the very early phase of the kinetic experiments is generally too limited to allow adequate description of either the distribution and elimination or the uptake, distribution and elimination in case of i.v. or oral application, respectively. The lack of a sufficient number of data-points may have led to the vast

The IISI Europe Natural Toxin Task Force supported the workshop at which this work was presented. Industry members of this task force are Groupe Danone, Kraft Foods, Nestlé, Numico, RHM Technology, and Swiss Quality Testing Services (SQTS). For further information about ILSI Europe, call +32 27710014 or email info@ilsieurope.be. The opinions expressed herein are those of the authors and do not necessarily represent the views of ILSI Europe.

Correspondence: Daniel R. Dietrich. E-mail: Daniel.Dietrich@uni-konstanz.de 
Table I. Kinetic parameters in orally (gavage) OTA-exposed (single application) humans and animals.

\begin{tabular}{|c|c|c|c|c|c|c|c|c|}
\hline Species & Sex & $\begin{array}{l}\text { Application } \\
\text { format }\end{array}$ & $\begin{array}{l}\text { Abs. } \\
(\%)\end{array}$ & $\begin{array}{l}\mathrm{t}_{1 / 2 \alpha} \\
(\mathrm{h})\end{array}$ & $\begin{array}{l}\mathrm{t}_{1 / 2 \beta} \\
(\mathrm{h})\end{array}$ & $\begin{array}{c}\text { Clearance } \\
\left(\mathrm{ml} \mathrm{h}^{-1} \mathrm{~kg}^{-1}\right) \\
\end{array}$ & Model proposed & Ref. \\
\hline Human & $\mathrm{m}$ & Ethanol/Saline & $>85.5$ & 20.13 & 853.2 & 0.0905 & 2-Compartment open & 1 \\
\hline Monkey (M. mulata) & $\mathrm{m}$ & $\mathrm{NaHCO}_{3}$ & 57 & n.r. & 510 & 0.18 & 1-Compartment (1st-order) & 2 \\
\hline Monkey (M. mulata) & $\mathrm{f}$ & $\mathrm{NaHCO}_{3}$ & 57 & n.r. & 510 & 0.18 & 1-Compartment (1st-order) & 2 \\
\hline Rat (F344) & $\mathrm{m}$ & Corn oil & n.r. & n.r. & 224 & n.r. & 1-Compartment (1st-order) & 3 \\
\hline Rat (F344) & $\mathrm{f}$ & Corn oil & n.r. & n.r. & 231 & n.r. & 1-Compartment (1st-order) & 3 \\
\hline Rat (Wistar) & $\mathrm{m}$ & $\mathrm{NaHCO}_{3}$ & 44 & n.r. & 120 & 0.91 & 2-Compartment open & 2 \\
\hline Rat (Sprague-Dawley) & $\mathrm{m}$ & $\mathrm{NaHCO}_{3}$ & n.r. & 2.1 & 57.8 & n.r. & 2-Compartment open & 4 \\
\hline Mouse (NIH-Bethesda) & $\mathrm{m}$ & $\mathrm{NaHCO}_{3}$ & 97 & n.r. & 39 & 6.1 & 2-Compartment open & 2 \\
\hline Fish (C. carpio) & n.r. & $\mathrm{NaHCO}_{3}$ & 1.6 & n.r. & 0.68 & 58 & 2-Compartment open & 2 \\
\hline Quail (C. coturnix) & $\mathrm{m}$ & $\mathrm{NaHCO}_{3}$ & 62 & n.r. & 6.7 & 57 & 2-Compartment open & 2 \\
\hline Quail (C. coturnix) & $\mathrm{f}$ & $\mathrm{NaHCO}_{3}$ & 62 & n.r. & 6.7 & 57 & 2-Compartment open & 2 \\
\hline
\end{tabular}

m: male; f: female; n.r.: not reported; 1: (Studer-Rohr et al. 2000); 2: (Hagelberg et al. 1989); 3: (Zepnik et al. 2003); 4: (Galtier et al. 1979); 5: (Stander et al. 2001); 6: (Hult et al. 1979); 7: ( $\mathrm{Li}$ et al. 1997); 8: (Galtier et al. 1981); 9: (Sreemannarayana et al. 1988).

Table II. Kinetic values in i.v. OTA-exposed (single application) animals.

\begin{tabular}{|c|c|c|c|c|c|c|c|}
\hline Species & Sex & $\begin{array}{c}\text { Application } \\
\text { format }\end{array}$ & $\begin{array}{c}t_{1 / 2 \alpha} \\
(\mathrm{h}) \\
\end{array}$ & $\begin{array}{l}\mathrm{t}_{1 / 2 \beta} \\
(\mathrm{h})\end{array}$ & Clearance $\left(\mathrm{ml} \mathrm{h}^{-1} \mathrm{~kg}^{-1}\right)$ & Model proposed & Ref. \\
\hline Monkey (M. mulata) & $\mathrm{m}$ & $\mathrm{NaHCO}_{3}$ & n.r. & 840 & 0.17 & 2-Compartment open & 2 \\
\hline Monkey (M. mulata) & $\mathrm{f}$ & $\mathrm{NaHCO}_{3}$ & n.r. & 840 & 0.17 & 2-Compartment open & 2 \\
\hline Monkey (C. aethiops) & $f$ & $\mathrm{NaHCO}_{3}$ & $58-72$ & $462-495$ & 0.22 & 2-Compartment open & 5 \\
\hline Pig & n.r. & $\mathrm{NaHCO}_{3}$ & n.r. & 150 & 1.5 & 2-Compartment open & 6 \\
\hline Pre-ruminant calves & n.r. & $\mathrm{NaHCO}_{3}$ & 1.8 & 77 & n.r. & 2-Compartment open & 9 \\
\hline Rat (Sprague-Dawley) & f & Ethanol/Saline & 2.7 & 103 & 3.11 & 2-Compartment open & 7 \\
\hline Rat (Sprague-Dawley) & $\mathrm{m}$ & $\mathrm{NaHCO}_{3}$ & 1.5 & 53.3 & n.r. & 2-Compartment open & 4 \\
\hline Rat (Wistar) & $\mathrm{m}$ & $\mathrm{NaHCO}_{3}$ & n.r. & 120 & 0.92 & 2-Compartment open & 2 \\
\hline Mouse (NIH-Bethesda) & $\mathrm{m}$ & $\mathrm{NaHCO}_{3}$ & n.r. & 48 & 6.1 & 2-Compartment open & 2 \\
\hline Rabbit & n.r. & $\mathrm{NaHCO}_{3}$ & 1.9 & 8.2 & n.r. & 2-Compartment open & 8 \\
\hline Fish (C. carpio) & n.r. & $\mathrm{NaHCO}_{3}$ & - n.r. & 8.3 & 57 & 2-Compartment open & 2 \\
\hline Quail (C. coturnix) & $\mathrm{m}$ & $\mathrm{NaHCO}_{3}$ & n.r. & 12 & 86 & 2-Compartment open & 2 \\
\hline Quail (C. coturnix) & $\mathrm{f}$ & $\mathrm{NaHCO}_{3}$ & n.r. & 12 & 86 & 2-Compartment open & 2 \\
\hline Chicken & n.r. & $\mathrm{NaHCO}_{3}$ & 0.5 & 4.1 & n.r. & 2-Compartment open & 8 \\
\hline
\end{tabular}

m: male; f: female; n.r.: not reported; 1: (Studer-Rohr et al. 2000); 2: (Hagelberg et al. 1989); 3: (Zepnik et al. 2003); 4: (Galtier et al. 1979); 5: (Stander et al. 2001); 6: (Hult et al. 1979); 7: (Li et al. 1997); 8: (Galtier et al. 1981); 9: (Sreemannarayana et al. 1988).

differences in elimination half-life $\left(t_{1 / 2 \beta}\right)$ reported for rats as well as in the, probably erroneous, assumption of a one-compartment open model such as in the monkey and the rat (Table I). Despite the fact that most authors used the same or similar mathematical calculations for the proposed one-or twocompartment open models, lack of raw data for inclusion in various dose-response models, prevents insertion of the raw data into one or several mathematical calculations and thus the direct comparison of the kinetic data published.

Consequently some of the differences in kinetic data reported in the literature for the same species, sex and type of application (e.g. the rat) may stem from differences in calculation, the dose-response model assumed or the limitations of the low number of time-points analysed rather than from real biological differences, e.g. sex, strain or dose and dose-format used. Other factors, which may also play a role, are discussed below. Most of the kinetic data available to date, (see Tables I and II), whether generated using i.v. or oral application, suggest the presence of a two-compartment open model, with blood representing the central compartment and all other organs (poorly or highly perfused) representing the peripheral compartment. It is of importance to state here that most kinetic models, even when calculating with measured OTA concentrations in the organs, did not consider the presence of active OTA transport beyond the uptake kinetics from the gastro-intestinal tract. The presence or absence and number of potential OTA transporters in the respective organs of the peripheral compartment, however, may additionally contribute to major differences in distribution and elimination kinetics in the various species investigated to date and possibly also between sexes. Consequently the "peripheral compartment" would have to be split into at least two compartments: One compartment with organs with a capacity for active OTA transport 
in addition to passive diffusion; and a further compartment where OTA gains entry into the organs via passive diffusion only. Indeed, it is important to understand that the elimination rate constant $\beta$ in the previously published two-compartment open model, in actuality, is a hybrid constant encompassing the elimination rate from the central as well as the peripheral compartment(s).

Thus, despite the fact that most current kinetic models calculated with a two-compartment open model, future models would have to consider threeor possibly even a four-compartment open model as more sophisticated predictors of the toxicokinetic properties of OTA in humans and other species. The latter models thus may then also provide a better insight, via more detailed understanding of the organ specific uptake and elimination constants, into the dynamically relevant OTA concentrations in situ and therefore allow better risk extrapolation and assessment. Indeed, the distribution of the raw data of all species tested to date for additional toxicokinetic analyses using more complex models would allow the implementation of these data in more sophisticated pharmacologically-based pharmacokinetic models or calculations with two-, three- or fourcompartment open models. This would certainly enhance trust in the current kinetic database and possibly provide more insight while preventing the repetition of expensive studies.

Although, the presently available analyses of the kinetic parameters (Tables I and II) derived from the two-compartment open model suggest that they do not fully represent reality, they certainly allow for an estimation of the real situation and thus enable deduction of some main observations while pointing to some open questions. The comparison of elimination half-lives obtained after i.v. or oral application in various species do demonstrate differences in some cases. Yet the overall impression is that these differences most likely are not factual differences but rather occur as a function of the amount of detectable OTA present in the plasma. Indeed, the i.v. application resulted in $100 \%$ availability of the OTA, thus allowing for longer and better detection of OTA in the plasma. In contrast, oral applications, judged by area-underthe-curve (AUC) comparison between oral and i.v. application (Hagelberg et al. 1989), resulted in a bioavailability between 1.6 and $97 \%$ of the dose, depending on the species. Hence, the observed differences in elimination half-life between oral and i.v. application routes most likely stem primarily from analytical limitations rather than from real biological effects. This observation is also corroborated by the fact that the calculated plasma clearance rates (Tables I and II) are comparable if not identical in the two application formats (i.v. vs. p.o.) and thus independent of the form of OTA application.

Further contributing factors, such as the main route of OTA excretion, may also play a large role. Following oral dosing OTA has been determined to be absorbed rapidly both from the stomach and the small intestine in rats and mice. Absorption from the jejunum can take place even against a concentration gradient (Kumagai and Aibara 1982), suggesting the presence of organic anion transporters with the capacity to transport OTA. Following absorption, OTA undergoes a certain degree of first-pass effect in all species. Factors such as serum protein binding (Hagelberg et al. 1989, Kuiper-Goodman and Scott 1989), and hence, freely-available OTA, the primary route of excretion (renal versus faecal), as well as potentially occurring enterohepatic recirculation and/or reabsorption of OTA, e.g. by the kidney, are then important for the elimination half-life of OTA. Sreemannarayana and co-workers (1988) observed a single protracted secondary peak in their analysis of serum OTA concentrations in calves following oral OTA administration and suggested this to be due to a superimposition of biliary OTA recycling on the overall elimination process. Similar observations have been made by other authors in rats and mice (Fuchs and Hult 1992, Fuchs et al. 1988, Roth et al. 1988). The reabsorption of OTA by the kidney has also been proposed to facilitate the residual persistence of the toxin and hence the observed renal toxicity in rodents (Albassam et al. 1987, Stein et al. 1985). In vivo metabolism of OTA in a number of species including rodents (Størmer et al. 1981) and ruminants (Pitout 1969) has been shown to yield predominantly the non-toxic congener ochratoxin- $\alpha(\mathrm{OT}-\alpha)$, i.e. the isocoumarin moiety lacking the phenylalanine group (see Figure 1).

This metabolic peptide bond cleavage appears to be a function of the intestinal tract as little conversion of the parent compound has been observed in either kidney or liver (Galtier et al. 1979, Hansen et al. 1982). Other detected metabolites include the $\mathrm{R}$ and $\mathrm{S}$ epimers of 4-hydroxy-OTA (4-OH-OTA). The prevalence of each of these epimers appears to be species-dependent, with for example 4R-OH-OTA predominating in human and rat microsomal systems (Størmer et al. 1981) and the $4 \mathrm{~S}-\mathrm{OH}$ variant being more commonly produced by pig liver microsomes. Størmer and co-workers also reported rabbit microsomes to produce 10-hydroxy-OTA. Both biliary and renal routes are involved in the excretion of OTA by rats and mice, with the biliary route predominating (Kuiper-Goodman and Scott 1989, Li et al. 1997, Moroi et al. 1985, Støren et al. 1982). Thus the observed shorter elimination half-lives in orally OTA dosed rats and mice and possibly quail and fish (Tables I and II) may partially be a reflection 


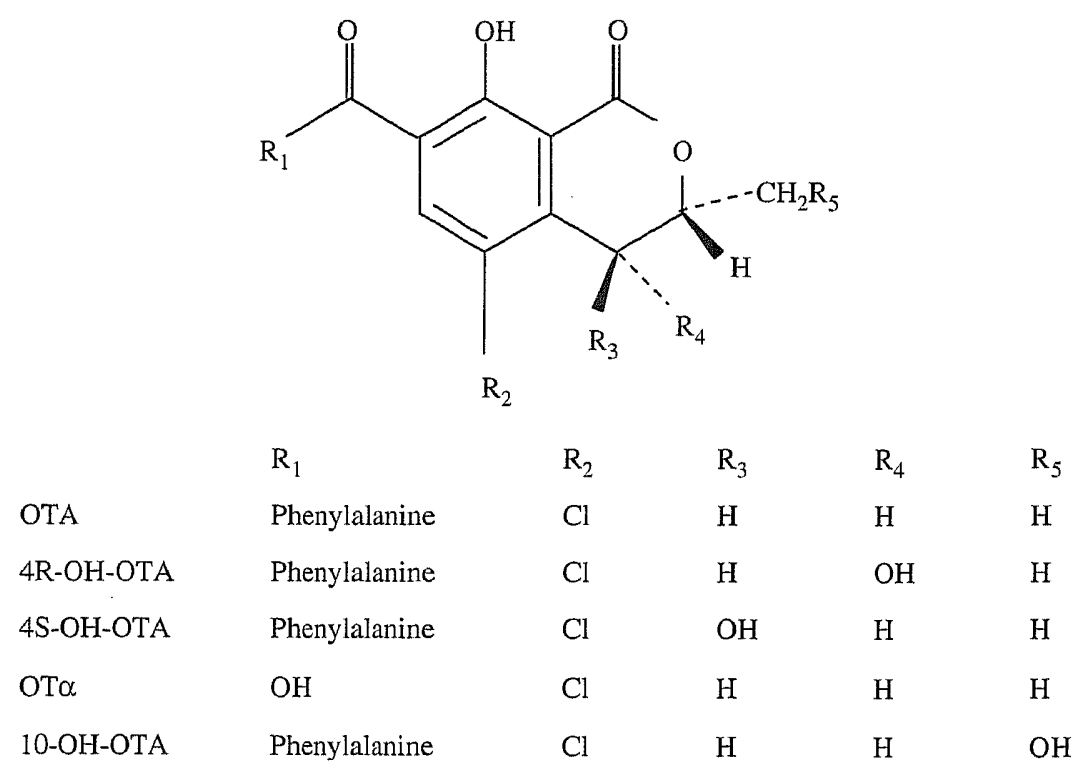

Figure 1. Chemical structures of OTA and some of its metabolites.

of the first-pass effect with primary biliary and subsequent faecal excretion. This would have as consequence that less OTA was detectable in the plasma at the later time points in the study.

In contrast, in humans and non-human primates (rhesus and vervet monkeys), predominantly parent compound is excreted and the primary route of excretion is via the kidney (Hagelberg et al. 1989, Stander et al. 2001, Studer-Rohr et al. 2000). Thus the observed differences in elimination half-lives observed after p.o. and i.v. application in the rhesus monkey is unlikely to stem from excretion pathway differences but appears primarily to stem from a rapid metabolic conversion of OTA to OT $\alpha$ as a result of the first-pass effect with consequently a more limited detection of the parent compound in the plasma during the course of the experiment. Consequently for risk assessment purposes, the i.v. elimination half-life appears to be more relevant as it reflects more precisely the conditions critical for potential renal changes than do oral elimination halflives, which by definition, also incorporate many other elimination constants, e.g. metabolism and other excretion routes. It is important to note here that the only human study carried out to date demonstrated that nearly $93 \%$ of the oral dose (dissolved in ethanol/saline) was observed in the blood at $8 \mathrm{~h}$ post exposure, thus suggesting that nearly $100 \%$ of the OTA was biologically available and little or none was metabolically converted during the first-pass effect. This may be due to the limited amount of freely-available (unbound) OTA, i.e. the high protein binding of OTA as already reported by Hagelberg and co-workers (1989) and as discussed below.
Despite the fact that Hagelberg and colleagues (1989) stated that they did not observe sex differences amongst the species they had investigated (rhesus monkeys, quail), experiments with SpragueDawley and F344 rats suggest that sex differences may exist. Indeed, the comparison of i.v. application of OTA in male and female Sprague-Dawley rats (Galtier et al. 1979, $\mathrm{Li}$ et al. 1997) suggest a longer distribution $\left(t_{1 / 2 \alpha}\right)$ and elimination half-life $\left(t_{1 / 2 \beta}\right)$ in females than in males (see Table II). Although not as unambiguous as the i.v. experiments, similar findings were reported by Zepnik and co-workers (2003) in male and female F344 rats gavaged with OTA in corn oil (see Table I). These reports thus raise several questions:

- Are sex differences also present in other species, e.g. mice?

- Are these differences a function of sex differences in protein binding and/or the presence/absence of OTA transporters in the respective organs?

- If female rats demonstrate a longer elimination half-life than male rats while being much more refractive to OTA, does this imply that it is not the continuous exposure to a given level of OTA but rather the repeated intermittent peak exposures to OTA, as suggested by O'Brien and coworkers (2001), that provide for the generation of a dedifferentiated renal cell population and thus giving rise to renal tumours?

\section{Protein binding}

Plasma protein binding, or more precisely the fraction of unbound and thus freely filterable 
proportion of OTA, is most likely one of the primary decisive factors in determining the half-life of OTA in any given species, and hence the susceptibility of that species to renal damage. Several studies have shown that OTA has an extremely high affinity for serum albumin and other macromolecules in the blood (Galtier 1978, Galtier et al. 1981, Hult and Fuchs 1986). In their comparison amongst species, Hagelberg and co-workers determined that humans and rats have a comparable unbound fraction of total OTA in the plasma $\left(f_{0}=0.02 \%\right)$, followed by monkey $\left(f_{o}=0.08 \%\right)$, mouse and pig $\left(f_{o}=0.1 \%\right)$, quail $\left(f_{o}=0.2 \%\right)$ and finally carp $\left(f_{o}=22.00 \%\right)$. The comparison of plasma protein binding affinity constants and calculated binding sites, demonstrated that, while the rat plasma protein had an approximately 40-fold higher affinity for OTA, the number of binding sites were 8-fold higher in the human. Despite sometimes up to 20 -fold higher OTA protein affinity constants, e.g. in the case of the mouse, most species investigated had a decisively lower number of binding sites than the human. Il'ichev et al. (2002) described high-affinity binding of OTA dianions, to human serum albumin (HSA). Using recombinant fractions of HSA, the authors concluded that HSA has at least two unique binding sites, each of which can accommodate one dianion. The highest affinity binding site was determined to be subdomain IIA of HSA, however subdomain IIB and domain I were determined to be critical for the integrity of this binding site. Interestingly aspartame could not displace OTA from these binding sites.

Thus it appears that while a high OTA binding affinity may provide for tighter binding, i.e. less likelihood of OTA release once bound, it is the number of binding sites that primarily determines the "reservoir" of OTA that can be released over a protracted time-period thus promoting a continuous availability of OTA. Unfortunately, no differentiation was made between the sexes of the species investigated therefore sex differences in binding quality and in quantity of plasma protein was not deducible. It has been suggested that the bond with plasma albumin, as one of the major plasma proteins, results in the generation of a mobile reservoir of OTA, which can be slowly released and hence rendered bioavailable over extended periods of time and furthermore, retard the elimination of OTA from the body. Kumagai (1985) provided further evidence for this with the demonstration that the half-life of OTA in albumin-deficient rats is much shorter than in normal rats. Moreover, this author also demonstrated 20-70-fold higher concentrations of OTA in the bile and urine of albumin-deficient rats than in the control cohort. These observations demonstrate the importance of plasma/protein binding for the biological half-life of OTA.
Renal proteins have also been demonstrated to have a strong affinity for OTA. Heussner et al. (2002) have reported outstanding species-dependent differences in the binding characteristics of OTA to proteins present in renal cortical homogenates from various species (pig, mouse, rat, and human of both sexes). Using a modification of a classical receptor-binding assay, these authors described the presence of at least one homogeneous OTA-binding component. This component appeared to have low affinity but high capacity for ${ }^{3} \mathrm{H}-\mathrm{OTA}$, which could be competed by a range of substances known to have affinity for steroid receptors and/or for various organic anion transporters previously reported to be responsible for the transport of OTA (Tsuda et al. 1999).

Based on the pattern of protein binding competition, the authors suggested that this binding component does not belong to the organic anionic transporters (see below) previously described. However, human renal proteins consistently displayed a 2-20-fold higher OTA binding capacity than any of the other species tested. The generated binding-capacity ranking of human $>$ rat $>$ pig $\geq$ mouse correlates well with the biological halflives determined by other authors (human $>$ rat $\geq$ pig $>$ mouse) and also with the toxicity ranking for experimental animals in vivo. Furthermore, sex differences in relative OTA binding were observed in the rat, with males having an approximately 3 -fold higher binding affinity for OTA than females (Heussner et al. 2002). Sex differences were also apparent with regard to the renal protein binding sites calculated, although the high standard deviations, which probably resulted from inter-individual variation, indicate that these values cannot be taken as definitive. Despite this, human renal proteins provided for the highest number of binding sites and mice the lowest, whereas female human renal proteins possessed a 5 -fold higher number of binding sites than male human renal proteins.

\section{Transport proteins}

In an aspect closely related to protein binding, the marked differences in the relative sensitivities of various species and sexes to OTA may also be partially governed by variations in the transporter complements of renal cells from different species. These could play a role in determining OTA accumulation in sensitive cells. Furthermore, species-and sex-specific expression of OTA-specific transporters could modulate organ and cellular OTA concentrations and kinetics and therefore have a direct influence on the toxicodynamics.

OTA has indeed been suggested to be a substrate for the family of organic anion transporter 
proteins (OATS). The best characterized of these is the SLC22A (formerly OAT1) family, made up of several splice variants from the same gene (Russel et al. 2002). This transporter family has extremely wide substrate specificity and has been found in numerous organ systems including the kidney. Other transporters include the members of the slc22a (formerly OATS) grouping, which are the rodent equivalents of the human proteins. Excellent, detailed reviews of the organic anion transporters are available (Masereeuw and Russel 2001, Russel et al. 2002) and further information on nomenclature can be obtained from the internet pages of the Human Gene Nomenclature Committee (HUGO) (http://www.gene.ucl.ac.uk/nomenclature) and the Rat Genome and Nomenclature Committee (RGNC) (http:// rgnc.gen.gu.se).

Which specific transporters are responsible for OTA transport, remains a matter of debate. Sokol and colleagues (1988) and Gekle and co-workers (1994) have reported transport to occur solely via the PAH transport pathway in rabbit renal basolateral membrane vesicles and opossum kidney cells, respectively, Groves et al. (1998) have reported that uptake occurs via a combination of passive diffusion and/or non-specific binding and carrier-mediated processes in rabbit renal proximal tubules.

Whichever is the case, cellular accumulation probably plays an important role in OTA-mediated cytotoxicity. O'Brien and colleagues (2001) demonstrated that primary renal epithelial cells of human and porcine origin rapidly accumulate 10-15-fold more ${ }^{3} \mathrm{H}$-OTA than their continuous cell line counterparts. Intracellular accumulation of OTA has also been shown by other researchers (Schwerdt et al. 1998) and demonstrated to occur extremely rapidly, with a plateau-phase occurring within 1-2 min of OTA addition to cultures of renal cells from mice expressing the multispecific human organic anion transporters hOAT1 (OAT1, SLC22A6) and hOAT3 (OAT3, SLC22A8) (Jung et al. 2001) and in mouse proximal tubule cells stably transfected with hOAT4 (SLC22A11) (Babu et al. 2002).

Support for the possible involvement of transporters in the observed sex- and species-differences in OTA-mediated toxicity is also to be found in the observations of Buist and Klaassen (Buist 2002, Buist and Klaassen 2003) who demonstrated large sex-, age- and species-dependent variations in the expression levels of a number of organic anion transporters. Sex-specific differences in mRNA expression levels were observed for oat2 (Slc22a7) and oat3 (Slc22a8) but not for oat1 (Slc22a6). If intracellular OTA accumulation and/or excretion are indeed a function of the presence or absence of the organic anion transporters, then a closer analysis of these proteins including expression levels and species- and substrate-specificity is required to better understand organ specific uptake and elimination kinetics and thus dynamics. Indeed, as sex differences have been suggested earlier from in vivo oral and i.v. kinetic studies, these observed sex differences may well stem from sex- and agedependent expression of transporters capable of transporting OTA. Understanding of the importance of these transporters would allow the generation of more detailed species-, sex- and organ-specific distribution and elimination constants and thus improve the base data set prerequisite for a more sophisticated pharmacologically-based pharmacokinetic model or at least for the generation of a three- or four-compartment open model.

\section{Implications for toxicity/carcinogenicity and human risk assessment}

O'Brien and co-workers (2001) and Heussner and colleagues (2000) investigated the cytotoxic effects of OTA in primary human, porcine and rat renal epithelial cells as well as continuous epithelial cell lines from rat (NRK-52E) and pig (LLC-PK1). In these studies, primary cells of human origin were reported to be the most sensitive cell type to the antiproliferative/cytotoxic effects of OTA, with a slight tendency for an even higher sensitivity of cells from female donors (significant reduction in cell numbers was apparent after 48-h exposure $1 \mathrm{nM}$ OTA). Interestingly, approximately $15 \%$ of each cell type survived exposure to even extremely high $(\geq 100 \mu \mathrm{M})$ concentrations of OTA over extended time periods $(\leq 96 \mathrm{~h})$ and could be demonstrated to re-enter the cell cycle and proliferate following removal of the toxin (Dreger et al. 2000, O'Brien et al. 2001, Wolf et al. 2002). The authors suggested the existence of an OTA-resistant, possibly apoptotic-defective, sub-population of cells. In the same study (O'Brien et al. 2001), the NRK-49F renal fibroblast cell line was shown to be relatively insensitive to the antiproliferative/cytotoxic effects of OTA, despite accumulating OTA concentrations comparable with their epithelial counterparts, NRK-52E. Similar observations of OTA resistance have been made for primary human fibroblasts (O'Brien, unpublished observation). This supports the author's hypothesis that the progressive fibrosis characteristic for BEN could arise through a cytotoxic/cytostatic effect of OTA in renal epithelial cells, coupled with continued fibroblast proliferation, resulting in progressive replacement of healthy, functional tissue with fibroblasts.

The exposure scenario described above, i.e. continuous intermittent peak exposures to OTA rather than a constant OTA concentration appears more likely to be that which promotes the generation 
of disease (e.g. BEN or UT in humans). Indeed, the present data suggest that despite a longer half-life, female rats are more refractive to OTA renal carcinogenesis than their male counterparts. A similar situation may be found if male and female mice are compared with regard to their kinetic properties. However, when comparing the mouse (Bendele et al. 1985) and rat (Boorman 1989) cancer bioassays, the dose that produced a $28 \%$ tumour incidence in male mice was approximately 20 -fold higher than the dose that produced a $60 \%$ tumour incidence in F344 male rats. In view of the faster elimination of OTA (high plasma clearance, low $t_{1 / 2 \beta}$ ) (Hagelberg et al. 1989) the question may be raised whether or not a chronic and biologically available OTA plasma level superimposed by continuous intermittent peak concentrations is necessary to induce the required cytotoxic/mitogenic stimulus in order to induce renal tumours. If this indeed were the case, the oral gavage of the mice (intermittent peak concentrations) and the chronic dosing offset by a relatively rapid elimination would explain why such a high dose of OTA was required to induce tumours in the male mice. Conversely, this could mean that the long half-life, high plasma protein and renal protein binding as well as the high oral bioavailability of OTA, when given in alcohol, represents a much higher risk for humans exposed to OTA continuously and intermittently than assumed beforehand. Unfortunately, current risk assessment schemes are primarily focused on the determination of a tolerable daily intake (TDI) broken down over all potential sources of intake and calculated to the respective average daily consumption, and do not model continuous exposure to low concentrations, (e.g. via coffee, bread, meat, juice, fruits and nuts) with intermittent peak exposures (e.g. via wine, beer).

\section{Open questions and conclusions}

The previous paragraphs clearly demonstrate that species- and sex- differences need better understanding with regard to the interpretation of the kinetic data presently at hand. In this regard it would be preferable to produce one single re-analysis of the presently available raw data, such that the kinetic data from various sources are more readily comparable. Furthermore, none of the species analysed to date provide for a good surrogate species for the human especially as differences in parameters intrinsically underlying the overall kinetics are such that they cannot be directly extrapolated from any one species to the human. In view of this, the generally applied two-compartment open model is a close but yet a mere approximation of the real kinetic situation and lacks the fine detail required to understand the kinetics and subsequent dynamics in situ, e.g. at the level of the kidney. The current data suggest that some of the species- and sexdifferences may be potentially explained by species-, sex- and age-specific variations in the expression levels of proteins capable of active OTA transport. Such critical data have not as yet been published for the human but their availability in the future would certainly increase the validity of any risk assessment model used. However, even if a better kinetic model could be designed, a much more sophisticated doseresponse model for the risk assessment of OTA in humans, considering continuous exposure levels as well as superimposed intermittent peak concentrations, is vital.

\section{References}

Albassam MA, Yong SI, Bhatnagar R, Sharma AK, Prior MG. 1987. Histological and electron microscopic studies on the acute toxicity of ochratoxin A in rats. Veterinary Pathology 24:427-435.

Babu E, Takeda $M$, Narikawa $S$, Kobayashi $Y$, Enomoto A, Tojo A, Cha SH, Sekine T, Sakthisekaran D, Endou H. 2002. Role of human anion transporter 4 in the transport of ochratoxin A. Biochimica et Biophysica Acta 1590:64-75.

Bendele AM, Carlton WW, Krogh EB, Lillehoj EB. 1985. Ochratoxin A carcinogenesis in the (C57BL/\&J X $\left.\mathrm{C}^{\star} \mathrm{H}\right) \mathrm{F}_{1}$ mouse. Journal of the National Cancer Institute 75(4):733-742.

Boorman GA. 1989. Toxicology and carcinogenesis studies of ochratoxin A in F344/N rats. NTP Technical Report NTP TR 358.

Buist SCN. 2002. Gender-specific and developmental influences on the expression of rat organic anion transporters. Journal of Pharmacology and Experimental Therapeutics 301:145-151.

Buist SCN, Klaassen CD. 2003. Species and gender differences inorganic anion transporter (OAT) mRNA. Toxicological Sciences (The Toxicologist) 72(S-1):259.

Dreger S, O'Brien E, Satck M, Dietrich D. 2000. Antiproliferative effects and cell-cycle specific effects of ochratoxin $A$ in LLC-PK1, NRK-52E and porcine primary proximal kidney cells. Toxicological Sciences 54(1):170.

Fuchs R, Hult K. 1992. Ochratoxin A in blood and its pharmacokinetic properties. Food and Chemical Toxicology 30(3):201-204.

Fuchs R, Radic B, Paraica M, Hult K, Plestina R. 1988. Enterohepatic circulation of ochratoxin $\mathrm{A}$ in rats. Periodicum Biologorum. 90(1):39-42.

Galtier P. 1978. Contribution of pharmacokinetic studies to mycotoxicology - Ochratoxin A. Veterinary Science Communications 1:349-358.

Galtier P, Alvinerie M, Charpenteau JL. 1981. The Pharmacokinetic profiles of ochratoxin $A$ in pigs, rabbits and chickens. Food and Cosmetics Toxicology 19:735-738.

Galtier P, Charpenteau J-L, Alvinerie M, Labouche C. 1979. The pharmacokinetic profile of ochratoxin $A$ in the rat after oral and intravenous administration. Drug Metabolism and Disposition $7(6): 429-434$.

Gekle M, Mildenberger S, Freudinger R, Silbernagel S. 1994. The mycotoxin ochratoxin $\mathrm{A}$ impairs protein uptake in cells derived from the proximal tubule of the kidney (Opossum kidney cells). Journal of Pharmacology and Experimental Therapeutics 271:1-6. 
Groves CE, Morales M, Wright SH. 1998. Peritubular transport of ochratoxin $\mathrm{A}$ in rabbit renal proximal tubules. Journal of Pharmacology and Experimental Therapeutics 284(3):943-948.

Hagelberg S, Hult K, Fuchs R. 1989. Toxicokinetics of ochratoxin $A$ in several species and its plasma-binding properties. Journal of Applied Toxicology 9(2):91-96.

Hansen CE, Dueland S, Drevon CA, Stormer FC. 1982. Metabolism of ochratoxin A by primary cultures of rat hepatocytes. Applied Environmental Microbiology 43(6):1267-1271.

Heussner A, Stack M, Hochberg K, Dietrich D. 2000. Comparison of cytotoxic effects of ochratoxin A and B on human, rat and porcine renal cells. Toxicological Sciences 54(1): 170 .

Heussner AH, O'Brien E, Dietrich DR. 2002. Species- and sexspecific variations in binding of ochratoxin $A$ by renal proteins in vitro. Experimental Toxicologic Pathology 54:151-159.

Hult K, Fuchs F. 1986. Analysis and dynamics of ochratoxin A in biological systems. In: Steyn PS, Vleggaar $R$, editors. Mycotoxins and phycotoxins. Amsterdam: Elsevier Science Publishers, B.V. pp 365-376.

Hult $\mathrm{K}$, Hokby E, Hagglund U, Gatenbeck S, Rutqvist L, Sellyey G. 1979. Ochratoxin A in pig blood: Method of analysis and use as a tool for feed studies. Applied and Environmental Microbiology 38:772-776.

Il'ichev YV, Perry JL, Rüker F, Dockal M, Simon JD. 2002. Interaction of ochratoxin A with human binding sites localized by competitive interactions with native protein and its recombinant fragments. Chemico-Biological Interactions 141:275-293.

IPCS/WHO. 2005. Principles for modelling dose-response for the risk assessment of chemicals. http://www.who.int/ipcs/methods/ harmonization/dose_response/en/: WHO task group on environmental health criteria for principles for modelling dose-response for the risk assessment of chemicals.

Jung KY, Takeda $M$, Kim DK, Tojo A, Narikawa S, Yoo BS, Hosoyamada M, Cha SH, Sekine T, Endou H. 2001. Characterization of ochratoxin A transport by human organic anion transporters. Life Sciences 69:2123-2135.

Kuiper-Goodman T, Scott PM. 1989. Risk assessment of the mycotoxin ochratoxin A. Biomedical and Environmental Sciences 2(3):179-248.

Kumagai S. 1985. Ochratoxin A: Plasma concentration and excretion into bile and urine in albumin-deficient rats. Food and Chemical Toxicology 23(10):941-943.

Kumagai S, Aibara K. 1982. Intestinal absorption and secretion of ochratoxin A in the rat. Toxicology and Applied Pharmacology 64:94-102.

Li S, Marquardt RR, Frohlich AA, Vitti TG, Crow G. 1997. Pharmacokinetics of ochratoxin $A$ and its metabolites in rats. Toxicology and Applied Pharmacology 145:82-90.

Masereeuw R, Russel FGM. 2001. Mechanisms and clinical implications in renal drug excretion. Drug Metabolism Reviews 33(3\&4):299-351.
Moroi K, Suzuki S, Kuga T, Yamazaki M, Kanisawa M. 1985. Induction of ochratoxin A toxicity in mice treated with phenylalanine and Phenobarbital. Toxicology Letters 25:1-5.

O'Brien E, Heussner AH, Dietrich D. 2001. Species-, sex- and cell type specific effects of ochratoxin A and B. Toxicological Sciences 63:256-264.

Pitout MJ. 1969. The hydrolysis of ochratoxin A by some proteolytic enzymes. Biochemical Pharmacology 18:485-491.

Roth A, Chakor K, Creppy EE, Kane A, Röschenthaler R, Dirheimer G. 1988. Evidence for an enterohepatic circulation of ochratoxin A in mice. Toxicology 48:293-308.

Russel FGM, Masereeuw R, van Aubel RAMH. 2002. Molecular aspects of renal anionic drug transport. Annual Review of Physiology 64:563-594.

Schwerdt G, Freudinger R, Silbernagl S, Gekle M. 1998. Apical uptake of radiolabelled ochratoxin A into Madin-Darby canine kidney cells. Toxicology 131:193-202.

Sokol PP, Ripich G, Holohan PD, Ross CR. 1988. Mechanism of ochratoxin A transport in kidney. Journal of Pharmacology and Experimental Therapeutics 246(2):460-465.

Sreemannarayana O, Frohlich AA, Vitti TG, Maryuardt RR, Abramson D. 1988. Studies of the tolerance and disposition of ochratoxin A in young calves. Journal of Animal Science 66:1703-1711.

Stander MA, Nieuwouldt TW, Steyn PS, Shepard GS, Creppy EE, Sewram V. 2001. Toxicokinetics of ochratoxin A in vervet monkeys (Chercopithecus aethiops). Archives of Toxicology 75:262-269.

Stein AF, Phillips TD, Kubena LF, Harvey RB. 1985. Renal tubular secretion and reabsorption as factors in ochratoxicosis: Effects of probenicid on nephrotoxicity. Journal of Toxicology and Environmental Health 16:593.

Studer-Rohr J, Schlatter J, Dietrich DR, 2000. Intraindividual variation in plasma levels and kinetic parameters of ochratoxin $\mathrm{A}$ in humans. Archives of Toxicology 74(9):499-510.

Støren O, Holm H, Størmer FC. 1982. Metabolism of ochratoxin $A$ by rats. Applied and Environmental Microbiology 44(4):785-789.

Størmer FC, Hansen CE, Pedersen JI, Hvistendahl G, Aasen AJ. 1981. Formation of (4R)- and (4S)-4-hydroxyochratoxin A from ochratoxin A by liver microsomes from various species. Applied Environmental. Microbiology 42(6):1051-1056.

Tsuda $M$, Sekine $T$, Takeda $M$, Cha SH, Kanai $Y$, Kimura $M$, Endou H. 1999. Transport of ochratoxin A by renal multispecific organic anion transporter 1. Journal of Pharmacology and Experimental Therapeutics 289:1301-1305.

Wolf $\mathrm{P}$, O'Brien E, Heussner AH, Stack ME, Thiel R, Dietrich DR. 2002. Sex- and age- specific effects of ochratoxin A in primary human kidney cells (HKC). Toxicological Sciences 66(1-S):400.

Zepnik H, Völkel W, Dekant W. 2003. Toxicokinetics of the mycotoxin ochratoxin A in F344 rats after oral administration. Toxicology and Applied Pharmacology 192:36-44. 